\title{
Comparison of Variant Robust SCUC Models for Operational Security and Economics of Power Systems under Uncertainty
}

\author{
Bingqian $\mathrm{Hu}^{(1)}$, Lei $\mathrm{Wu}^{1}{ }^{(1)}$, Xiaohong Guan ${ }^{(2)}$, Feng Gao ${ }^{(2)}$, Qiaozhu Zhai ${ }^{(2)}$ \\ ${ }^{(1)}$ ECE Department, Clarkson University, Potsdam, NY 13699, USA
}

\begin{abstract}
In electricity market, security-constrained unit commitment (SCUC) utilizes the detailed market information submitted by market participants to provide a financially viable and physically feasible unit commitment solution for Independent system operators (ISOs)/regional transmission owners (RTOs). With the dramatic increase of renewable energy resources in recent years, the robust optimization approach has been recently explored for solving the SCUC problem with various uncertainties. This paper focuses on the following questions: How variant robust SCUC models in terms of different worst case definitions could impact operational security and economics of power systems under uncertainties? And what is the proper robust SCUC model that can meet specific market operation needs of ISOs/RTOs for effectively operating the system? Four different robust SCUC models are investigated, including: (1) Minimax load shedding robust model, (2) Minimax economic dispatch (ED) cost robust model, (3) Minimax variance robust model, and (4) Minimax regret robust model. Outer approximation (OA) and Benders' decomposition (BD) are used to solve the four robust models. Numerical case studies compare the performance of the four models and analyze their distinct characteristics for the market economic operation (such as locational marginal prices (LMP), regret costs, and base case costs) and system secure operation (such as load shedding (LS) quantities and Do-Not-Exceed (DNE) limits) of power systems under uncertainties.
\end{abstract}

Keywords: Renewable energy resource, restructured power markets, security-constrained unit commitment, uncertainty.

\section{NOMENCLATURE}

The symbols used in this paper are classified into indices, parameters, and decision variables as follows.

\section{Indices}

d Index for loads

$t \quad$ Subscript for time

w Index for wind farms

\section{Parameters}

$\boldsymbol{A}, \boldsymbol{B}, \boldsymbol{C} \quad$ Coefficient matrices of prevailing SCUC constraints

* Corresponding author. Tel.: +1 3152683914. E-mail address: 1wu@ clarkson.edu (Lei Wu) 


$\begin{array}{ll}\boldsymbol{c} & \text { Vector of incremental costs of generators } \\ \boldsymbol{D}^{b} & \text { Vector of forecasted injections } \\ \boldsymbol{\Omega} & \text { Uncertainty set } \\ \boldsymbol{N} & \text { Vector of no-load, startup, and shutdown costs of generators } \\ N T & \text { Number of hours } \\ \tilde{P}_{d t}, \tilde{P}_{f, w t} & \text { Variations of hourly loads and wind power outputs } \\ P_{d t}^{b}, P_{f, w t}^{b} & \text { Forecasts on hourly loads and wind power outputs } \\ \Delta_{d}, \Delta_{w} & \text { Budget limits of uncertain loads and wind power outputs } \\ \Delta \boldsymbol{P} & \text { Vector of corrective capacities of generators }\end{array}$

\section{Decision Variables}

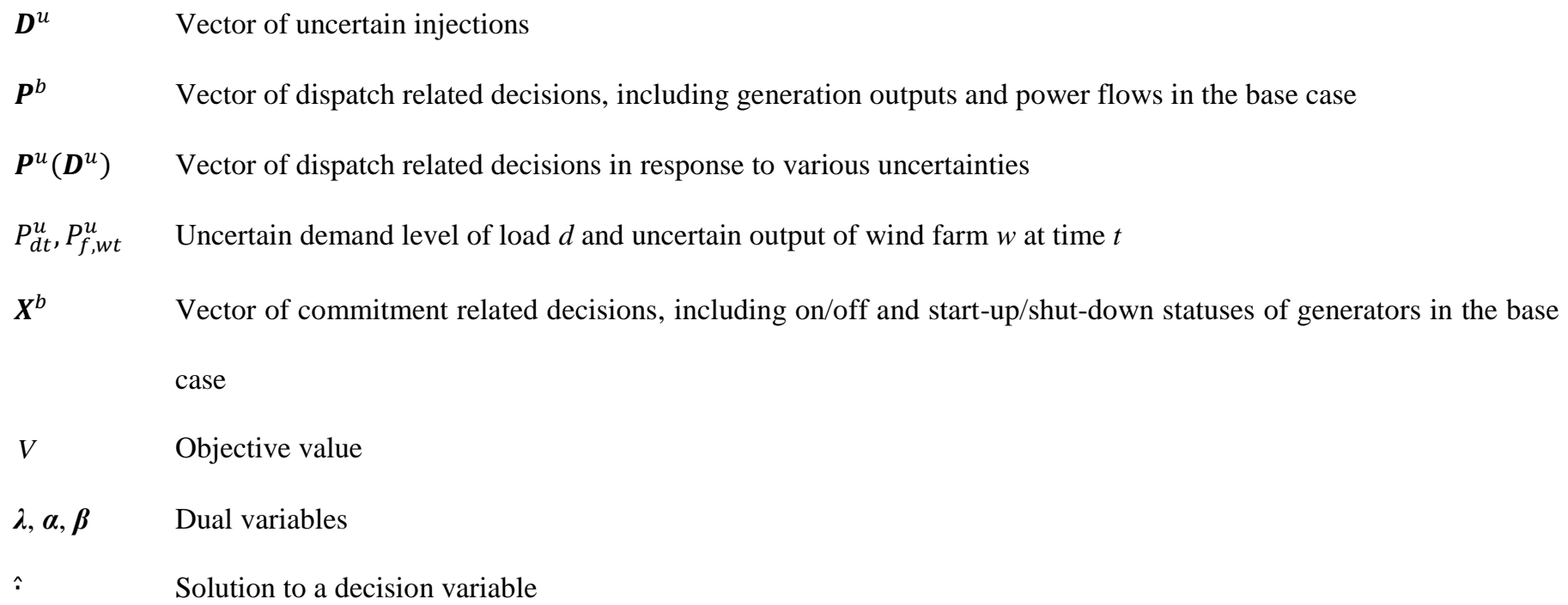

\section{INTRODUCTION}

SCUC is one of the most important operation planning tasks in power systems. In restructured power markets, SCUC is utilized by ISOs and RTOs to clear day-ahead/week-ahead electricity markets, with hourly forecasts on loads and renewable energy outputs over a certain time period. However, load and renewable generation are usually considered to be uncertain, and their real-time realizations could significantly deviate from the forecast values. Therefore, determining the optimal hourly unit commitment and generation dispatch solutions to satisfy actual demand and reliability requirements is a challenge for managing operational security and economics of power systems. Different approaches have been explored in literature to tackle this problem, including deterministic spinning reserve method [1], stochastic optimization method [2], and chance-constrained optimization method [3].

Robust optimization has recently gained substantial popularity as a modeling framework for mathematical optimization under 
parameter uncertainty [4], which provides an effective way for handling uncertainties in the real-time operation. Robust SCUC adopts deterministic uncertainty sets to represent uncertain factors, which are constructed via limited information on uncertain quantities, namely the mean together with a range of possible variations around the mean [5]. The basic idea of the robust SCUC is to identify the worst case of the system operation with respect to all possible uncertainty realizations, and use it to advise the day-ahead schedule for maintaining operational security and economics. Most robust SCUC models in literature are constructed based on the worst case ED cost. However, different worst-case definitions may derive different robust SCUC solutions, which in turn could impact operational security and economics of power systems under uncertainties. The motivation behind this paper is to investigate distinct performances of different robust SCUC models on the operational security and economics of power systems. This paper explores four robust SCUC models based on different worst case definitions as follows.

(1) The first model is the Minimax Load shedding (LS) Robust (MLR) SCUC model, in which the worst case refers to the largest LS quantity under uncertainties. In order to guarantee the operational security when real-time uncertainty realizations deviate from the forecasts, the day-ahead solution is adjusted to accommodate the largest LS scenario. The MLR model minimizes the day-ahead operation costs in the base case, while ensuring that the day-ahead solution could be adaptively and securely adjusted in response to any uncertainty realization. The basic idea of the MLR model is to adjust the base case UC and ED solutions, in order to minimize the worst case LS quantity when hourly load and wind power generation vary within their uncertain intervals. This model was studied in authors' previous work [6].

(2) The second model is the Minimax Economic dispatch cost Robust (MER) SCUC model, in which the worst case refers to the highest ED cost under uncertainties. MER minimizes the day-ahead UC cost plus the highest ED cost under uncertainty, while guaranteeing that the day-ahead solution could be adaptively and securely adjusted in response to any uncertainty realization. Compared with MLR, the major difference is that the objective function of the MER model is to minimize the UC cost of the base case plus the highest dispatch cost under uncertainties. That is, the worst case is defined as the highest dispatch cost in the MER model, instead of the largest LS quantity in the MLR model. [7] applied the MER model to coordinate wind and pumped-storage for handling wind generation uncertainties. [8] studied the MER model for handling uncertainties of wind energy and demand response. Exceptionally, [9] proposed a stochastic day-ahead scheduling of power systems with flexible resources, while considering corrective actions of thermal units, energy storage, and hourly demand response for managing the uncertainty of renewable energy sources. In addition, [10] considered the hourly coordination of wind power generation units and pumped-storage hydro generation for relieving variability and increasing dispatchability of wind energy.

(3) The third model is the Minimax operation cost Variance Robust (MVR) SCUC model, in which the worst case refers to the highest ED cost deviation under uncertainties, i.e., the difference between the highest and the lowest ED costs with respect to all possible uncertainty realizations. MVR minimizes the day-ahead UC cost plus the highest ED cost deviation, while 
ensuring that the day-ahead solution could be adaptively and securely adjusted in response to any uncertainty realization. System operators may not prefer a day-ahead SCUC schedule that derives too volatile real-time dispatch costs with respect to possible uncertainty realizations. Therefore, the key idea of the MVR model is to determine a robust base case SCUC solution that would minimize the difference between the highest and the lowest dispatch costs under uncertainties. That is, the MVR model would derive relatively stable dispatch cost with respect to different uncertainty realizations. [11] studied a minimax approach to reduce the cost variance with imperfect information on parameters. [12] derived robust estimators of correlation and covariance matrices for ensuring a high stability of statistical inferences when presumed distributions are perturbed. Currently, authors are not aware of any literature that applies the MVR model to SCUC problems.

(4) The fourth model is the Minimax Regret Robust (MRR) SCUC model, in which the worst case refers to the largest regret value under uncertainties. Regret is defined as the difference between the operation cost of the robust SCUC solution in response to a certain uncertainty realization and that of the SCUC solution by treating this realization as perfect information. MRR minimizes the day-ahead UC cost plus the largest regret value, while adaptively and securely adjusting the day-ahead SCUC solution in response to any uncertainty realization. In power systems operation, the ideal situation is that the system operators would know the perfect information on real-time uncertainties in the day-ahead timeframe, so that the day-ahead solution would exactly match the real-time situation. Thus, the MRR model adjusts the base case UC solution by minimizing the largest difference between the robust SCUC solution and the SCUC solution with perfect information, and in turn reduces the regret caused by various uncertainties in the real-time operation. [13] discussed a similar MRR SCUC model, while neglecting the base case robust dispatch decision and corrective actions of generators in response to uncertainties.

In this paper, all four robust SCUC models are solved by BD [14], which decomposes the original problem into a master UC problem for the base case and feasibility/optimality checking subproblems for uncertainty intervals. When violations are identified in subproblems, feasibility and/or optimality Benders cuts are generated and fed back to the master problem for further iterations. The modified IEEE 118-bus system is studied for exploring the impacts of variant robust SCUC models on the operational security and economics of power systems under uncertainty. The major contributions of the paper are twofold.

(1) This paper for the first time applies the MVR model to SCUC problems. In addition, although similar MER SCUC models and MRR SCUC models have been discussed in literature, most of them neglect the base case robust dispatch solution and corrective actions of generators in response to uncertainties. In this paper, all the four robust SCUC models are formulated as a two-stage structure, in which the first stage includes base case robust unit commitment and dispatch solutions, and the second stage considers dispatch adjustment in response to uncertainties. This is consistent with the day-ahead market clearing and the real-time operation, and guarantees the fair comparison among the four robust SCUC models.

(2) This paper provides a systematic comparison of the four robust SCUC models, including base case UC cost, base case dispatch 
cost, LS, scenario dispatch cost, locational marginal price, regret cost, Do-Not-Exceed (DNE) limit, and computational performance. DNE limit refers to the largest potential uncertainty handling capacity that a robust solution can accommodate without sacrificing system security requirement. Numerical studies show the impacts of variant robust SCUC models on the operational security and economics of power systems under uncertainty, and provide insights into the proper robust SCUC model that could meet specific market operation needs of ISOs/ RTOs for effectively operating power systems.

The rest of the paper is organized as follows. Sections 2-5 present the four robust SCUC models and their solution procedures, respectively. Section 6 applies the modified IEEE 118-bus system to compare the performance of the four robust SCUC models in details, and conclusions are drawn in Section 7.

\section{MINIMAX LOAD SHEDDING RobUST (MLR) SCUC}

\section{A. MLR SCUC Formulation}

The basic idea of the MLR model is to adjust the base case UC and ED solutions, in order to minimize the worst case LS quantity when hourly load and wind power generation vary within their uncertain intervals. Its compact matrix formulation is shown in (1).

$$
\begin{array}{ll}
\operatorname{Min}_{\boldsymbol{X}^{b}, \boldsymbol{P}^{b}} & \boldsymbol{N}^{T} \cdot \boldsymbol{X}^{b}+\boldsymbol{c}^{T} \cdot \boldsymbol{P}^{b} \\
\text { s.t. } & \boldsymbol{A} \cdot \boldsymbol{X}^{b}+\boldsymbol{B} \cdot \boldsymbol{P}^{b} \leq \boldsymbol{C} \cdot \boldsymbol{D}^{b} \\
& \boldsymbol{A} \cdot \boldsymbol{X}+\boldsymbol{B} \cdot \boldsymbol{P}^{u}\left(\boldsymbol{D}^{u}\right) \leq \boldsymbol{C} \cdot \boldsymbol{D}^{u}, \quad \boldsymbol{D}^{u} \in \boldsymbol{\Omega} \\
& \left|\boldsymbol{P}^{b}-\boldsymbol{P}^{u}\left(\boldsymbol{D}^{u}\right)\right| \leq \Delta \boldsymbol{P}, \quad \boldsymbol{D}^{u} \in \boldsymbol{\Omega} \\
\boldsymbol{\Omega}\left(P_{d t}^{b}, \tilde{P}_{d t}, P_{f, w t}^{b}, \tilde{P}_{w t}, \Delta_{d}, \Delta_{w}\right):=\left\{\sum_{t}\left|P_{d t}^{u}-P_{d t}^{b}\right| / \tilde{d}_{d t} \leq \Delta_{d}, \quad P_{d t}^{u} \in\left[P_{d t}^{b}-\tilde{P}_{d t}, P_{d t}^{b}+\tilde{P}_{d t}\right], \sum_{t}\left|P_{f, w t}^{u}-P_{f, w t}^{b}\right| / \tilde{P}_{w t} \leq \Delta_{w}, \quad P_{f, w t}^{u} \in\left[P_{f, w t}^{b}-\tilde{P}_{w t}, P_{f, w t}^{b}+\tilde{P}_{w t}\right]\right\}
\end{array}
$$

The objective in (1) is to minimize the UC $\operatorname{cost} \boldsymbol{N}^{T} \cdot \boldsymbol{X}^{b}$ and the dispatch $\operatorname{cost} \boldsymbol{c}^{T} \cdot \boldsymbol{P}^{b}$ for the base case, while guaranteeing that the base case UC and ED solutions could be adaptively and securely adjusted in response to various uncertainties. The first constraint set in (1) represents prevailing system and unit constraints in the base case, including power balance, ramp up/down, minimum up/down time, generation dispatch and reserve limits, and transmission network security constraints. The second constraint set includes power balance, power generation limits (restricted by the base case UC), and transmission network security constraints in response to uncertainties. The third constraint set represents that dispatch adjustments of units in response to uncertainties are restricted by corrective capacities $\Delta \boldsymbol{P}$ and dispatches in the base case $\boldsymbol{P}^{b}$. Corrective capacities $\Delta \boldsymbol{P}$ refer to the 10-minute spinning reserve capabilities of online generators.

The last constraint in (1) describes the uncertainty set. Uncertain intervals $\left[P_{d t}^{b}-\tilde{P}_{d t}, P_{d t}^{b}+\tilde{P}_{d t}\right]$ and $\left[P_{f, w t}^{b}-\tilde{P}_{f, w t}, P_{f, w t}^{b}+\tilde{P}_{f, w t}\right]$ are used to represent variations of hourly loads $P_{d t}^{b}$ and wind power generations $P_{f, w t}^{b}$. $P_{d t}^{u}$ and $P_{f, w t}^{u}$ represent possible load and wind generation realizations, which could take any value within their uncertain intervals, i.e., $P_{d t}^{u} \in\left[P_{d t}^{b}-\tilde{P}_{d t}, P_{d t}^{b}+\tilde{P}_{d t}\right]$ and $P_{f, w t}^{u} \in\left[P_{f, w t}^{b}-\tilde{P}_{f, w t}, P_{f, w t}^{b}+\tilde{P}_{f, w t}\right]$. Uncertainty budget constraints are applied to control 
that the total deviations of loads and wind outputs from their forecasts throughout the entire scheduling horizon cannot exceed the predefined budget limits $\Delta_{d}$ and $\Delta_{w}$, which take values between 0 and $N T$. As $\Delta_{d}$ and $\Delta_{w}$ increase, the size of the uncertainty set $\boldsymbol{\Omega}$ enlarges, and a larger total deviation from forecast values will be considered. Thus, the resulting robust SCUC solution would be more conservative and the system is protected against a higher degree of uncertainty. The detailed formulation of the MLR SCUC model could be referred to authors' previous works [6] and [15].

\section{B. MLR SCUC Solution Methodology}

BD is adopted to solve the MLR SCUC model (1). As shown in Fig. 1, the MLR model is solved by the following steps:

Step 1: Solve the master UC problem for the base case. The master UC problem (2) minimizes the operation cost of the base case, subject to the first constraint set in (1) and all feasibility Benders cuts obtained from subproblems.

$$
\begin{array}{ll}
\operatorname{Min}_{\boldsymbol{X}^{b}, \boldsymbol{P}^{b}} & \boldsymbol{N}^{T} \cdot \boldsymbol{X}^{b}+\boldsymbol{c}^{T} \cdot \boldsymbol{P}^{b} \\
\text { s.t. } & \boldsymbol{A} \cdot \boldsymbol{X}^{b}+\boldsymbol{B} \cdot \boldsymbol{P}^{b} \leq \boldsymbol{C} \cdot \boldsymbol{D}^{b} \text { and all feasibility Benders cuts }
\end{array}
$$

Step 2: Check feasibility by identifying the largest LS. With current solutions $\widehat{\mathbf{P}}^{b}$ and $\widehat{\mathbf{X}}^{b}$ from the master problem, the max-min problem (3) identifies the largest LS when hourly load and wind generation vary within the uncertainty set $\boldsymbol{\Omega}$.

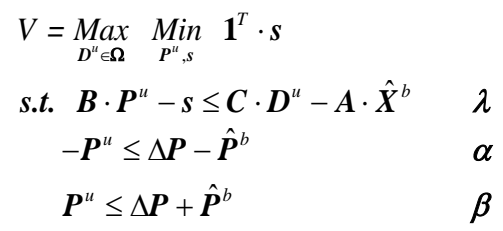

By adopting the duality theory of linear programming (LP) to transfer the inner minimization problem of (3) into an equivalent maximization problem, (3) can be converted into a single-level bilinear optimization problem (4), where $\lambda, \boldsymbol{\alpha}$, and $\boldsymbol{\beta}$ are dual variables of corresponding constraints in (3). The OA method [16] is adopted in this paper to solve the bilinear optimization problem (4), in which the bilinear term in the objective is linearly approximated around the current solution points and added to the OA formulation.

$$
\begin{aligned}
& V=\underset{\boldsymbol{D}^{u} \in \boldsymbol{\Omega}}{\operatorname{Max}}\left(\boldsymbol{C} \cdot \boldsymbol{D}^{u}-\boldsymbol{A} \cdot \hat{\boldsymbol{X}}^{b}\right)^{T} \cdot \boldsymbol{\lambda}+\left(\Delta \boldsymbol{P}-\hat{\boldsymbol{P}}^{b}\right)^{T} \cdot \boldsymbol{\alpha}+\left(\Delta \boldsymbol{P}+\hat{\boldsymbol{P}}^{b}\right)^{T} \cdot \boldsymbol{\beta} \\
& \text { s.t. } \quad \boldsymbol{B}^{T} \cdot \boldsymbol{\lambda}-\boldsymbol{\alpha}+\boldsymbol{\beta} \leq \mathbf{0}, \quad-\mathbf{1} \leq \boldsymbol{\lambda} \leq \mathbf{0}, \quad \boldsymbol{\alpha} \leq \mathbf{0}, \quad \boldsymbol{\beta} \leq \mathbf{0}
\end{aligned}
$$

If the largest LS quantity obtained from (4) is smaller than the predefined threshold, the current solution $\widehat{\boldsymbol{P}}^{b}$ and $\widehat{\boldsymbol{X}}^{b}$ is the final MLR SCUC solution. Otherwise, feasibility Benders cut (5) will be generated and fed back to the master problem (2) for seeking robust UC solutions that would alleviate security violations. The iterative procedure stops when the master problem solution satisfies all security violation checks. That is, no more feasibility cuts are generated in this step.

$-\lambda^{T} \cdot \boldsymbol{A} \cdot\left(\boldsymbol{X}^{b}-\hat{\boldsymbol{X}}^{b}\right)+(\boldsymbol{\beta}-\boldsymbol{\alpha})^{T} \cdot\left(\boldsymbol{P}^{b}-\hat{\boldsymbol{P}}^{b}\right)+\hat{V} \leq 0$ 


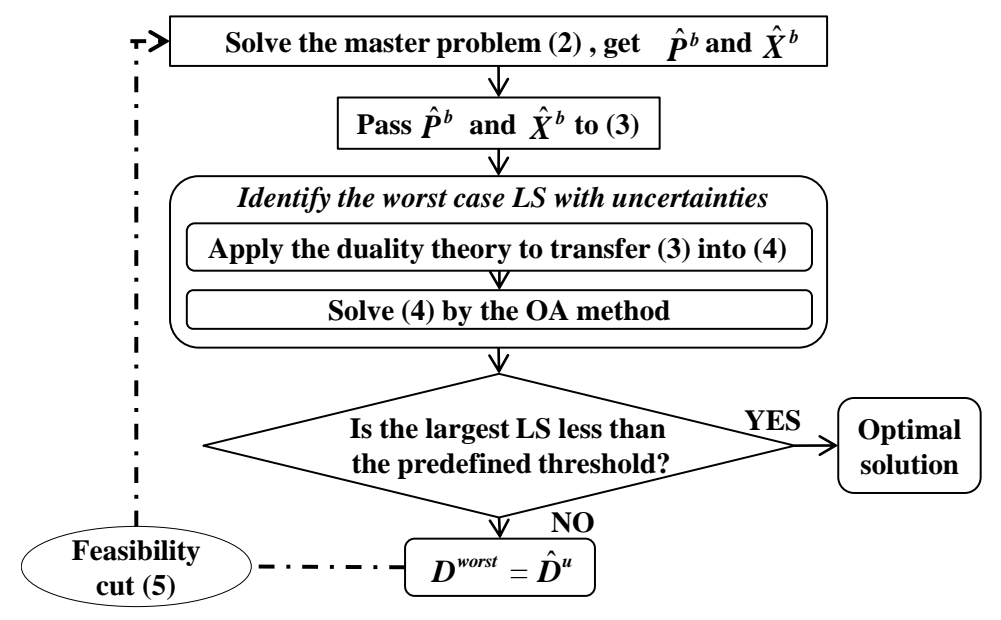

Fig. 1. Solution procedure of the MLR SCUC model

\section{Minimax ED COST RobUST (MER) SCUC}

\section{A. MER SCUC Formulation}

The MER SCUC model is presented in (6). Although it has the same set of constraints with the MLR model, the major difference is that the objective function of the MER model is to minimize the UC cost $\boldsymbol{N}^{T} \cdot \boldsymbol{X}^{b}$ of the base case plus the highest dispatch cost $\boldsymbol{c}^{T} \cdot \boldsymbol{P}^{u}\left(\boldsymbol{D}^{u}\right)$ under uncertainties. That is, the worst case is defined as the highest dispatch cost in the MER model, instead of the largest LS quantity in the MLR model.

$$
\begin{aligned}
& \underset{\boldsymbol{X}^{b}}{\operatorname{Min}}\left[\boldsymbol{N}^{T} \cdot \boldsymbol{X}^{b}+\underset{\boldsymbol{D}^{u} \in \Omega}{\operatorname{Max}} \operatorname{Min} \boldsymbol{P}^{u} \boldsymbol{c}^{T} \cdot \boldsymbol{P}^{u}\right] \\
& \text { s.t. } \boldsymbol{A} \cdot \boldsymbol{X}^{b}+\boldsymbol{B} \cdot \boldsymbol{P}^{b} \leq \boldsymbol{C} \cdot \boldsymbol{D}^{b} \\
& \boldsymbol{A} \cdot \boldsymbol{X}^{b}+\boldsymbol{B} \cdot \boldsymbol{P}^{u} \leq \boldsymbol{C} \cdot \boldsymbol{D}^{u} \\
& \left|\boldsymbol{P}^{b}-\boldsymbol{P}^{u}\right| \leq \Delta \boldsymbol{P}
\end{aligned}
$$

\section{B. MER SCUC Solution Methodology}

BD can also be adopted to solve the MER SCUC model. However, different from the MLR model solution procedure, as the second stage decision variables $\boldsymbol{P}^{u}$ are presented in the objective function, the solution procedure of the MER SCUC model would involve both feasibility and optimality checks. As shown in Fig. 2, the MER model is solved by the following steps:

Step 1: Solve the master UC Problem (7) for the base case, where $\theta$ is a non-negative ancillary variable to represent the lower bound of $\operatorname{Max}_{D^{u} \in \Omega^{M i n}} \operatorname{Pi}^{T} \cdot \boldsymbol{P}^{u}$.

$\min _{\boldsymbol{X}^{b}, \boldsymbol{P}^{b}} \boldsymbol{N}^{T} \cdot \boldsymbol{X}^{b}+\theta$
s.t. $\boldsymbol{A} \cdot \boldsymbol{X}^{b}+\boldsymbol{B} \cdot \boldsymbol{P}^{b} \leq \boldsymbol{C} \cdot \boldsymbol{D}^{b}$ and all feasibility/optimality Benders cuts

Step 2: Check feasibility by identifying the largest LS quantity. In this step, similar as (3)-(5) in the MLR model, the current solution $\widehat{\mathbf{P}}^{\mathrm{b}}$ and $\widehat{\mathbf{X}}^{\mathrm{b}}$ from the master problem is checked to identify the largest LS quantity when hourly load and wind generation 
vary within the uncertainty set $\boldsymbol{\Omega}$. If the largest LS is higher than the predefined threshold, the feasibility Benders cut (5) will be generated and fed back to the master problem (7), and go back to Step 1. Otherwise, go to Step 3.

Step 3: Check optimality by calculating the highest dispatch cost. If the base case solutions $\widehat{\boldsymbol{P}}^{b}$ and $\widehat{\boldsymbol{X}}^{b}$ pass the feasibility check in Step 2, they will be further evaluated in the optimality subproblem (8) to identify the highest dispatch cost, when hourly load and wind generation vary within the uncertainty set $\boldsymbol{\Omega}$. Similar as (3), (8) can be converted into a single-level bilinear optimization problem (9), where $\lambda, \boldsymbol{\alpha}$, and $\boldsymbol{\beta}$ are dual variables of corresponding constraints in (8). The OA method is again applied to solve the bilinear optimization problem (9).

If the optimal objective $\widehat{\boldsymbol{\omega}}$ is equal to $\hat{\theta}$ obtained in Step 1, the current solution $\widehat{\boldsymbol{P}}^{b}$ and $\widehat{\boldsymbol{X}}^{b}$ is the final optimal MER SCUC solution. Otherwise, an optimality Benders cut (10) will be generated and added to the next iteration of the master problem, for seeking robust UC solutions that would minimize the worst case dispatch cost. The iterative procedure stops when the master solution satisfies the optimality check. That is, no more optimality cuts are generated in this step.

$$
\begin{aligned}
& V=\underset{D^{u} \in \boldsymbol{\Omega}}{\operatorname{Max}} \underset{\boldsymbol{P}^{u}}{\operatorname{Min}} \boldsymbol{c}^{T} \cdot \boldsymbol{P}^{u} \\
& \text { s.t. } \boldsymbol{B} \cdot \boldsymbol{P}^{u} \leq \boldsymbol{C} \cdot \boldsymbol{D}^{u}-\boldsymbol{A} \cdot \hat{\boldsymbol{X}}^{b} \quad \boldsymbol{\lambda} \\
& -\boldsymbol{P}^{u} \leq \Delta \boldsymbol{P}-\hat{\boldsymbol{P}}^{b} \quad \boldsymbol{\alpha} \\
& \boldsymbol{P}^{u} \leq \Delta \boldsymbol{P}+\hat{\boldsymbol{P}}^{b} \quad \boldsymbol{\beta} \\
& V=\underset{\boldsymbol{D}^{u} \in \Omega}{\operatorname{Max}}\left(\boldsymbol{C} \cdot \boldsymbol{D}^{u}-\boldsymbol{A} \cdot \hat{\boldsymbol{X}}^{b}\right)^{T} \cdot \boldsymbol{\lambda}+\left(\Delta \boldsymbol{P}-\hat{\boldsymbol{P}}^{b}\right)^{T} \cdot \boldsymbol{\alpha}+\left(\Delta \boldsymbol{P}+\hat{\boldsymbol{P}}^{b}\right)^{T} \cdot \boldsymbol{\beta} \\
& \text { s.t. } \boldsymbol{B}^{T} \cdot \lambda-\alpha+\beta \leq c, \\
& \lambda \leq \mathbf{0}, \quad \alpha \leq \mathbf{0}, \quad \beta \leq \mathbf{0} \\
& \theta \geq \hat{V}-\lambda^{T} \cdot \boldsymbol{A} \cdot\left(\boldsymbol{X}^{b}-\hat{\boldsymbol{X}}^{b}\right)+(\boldsymbol{\beta}-\boldsymbol{\alpha})^{T} \cdot\left(\boldsymbol{P}^{b}-\hat{\boldsymbol{P}}^{b}\right)
\end{aligned}
$$

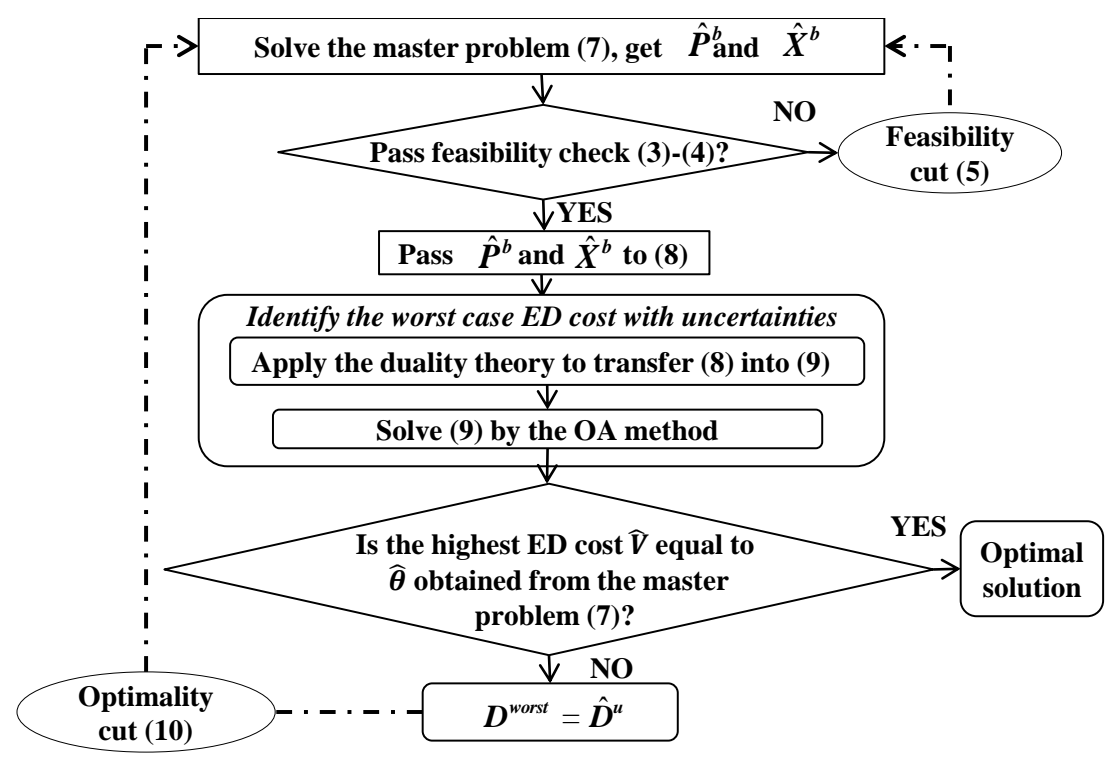

Fig. 2. Solution procedure of the MER SCUC model 


\section{MinimaX VARIANCE RobUSt (MVR) SCUC}

\section{A. MVR SCUC Formulation}

ISOs/RTOs may not prefer a day-ahead SCUC schedule that derives too volatile real-time dispatch cost with respect to possible uncertainty realizations. Therefore, the purpose of the MVR model is to determine a robust base case SCUC solution that would minimize the difference between the highest and the lowest dispatch costs under uncertainties. That is, the MVR model would derive relatively stable dispatch cost with respect to different uncertainty realizations.

The MVR SCUC model (11) is to minimize the UC cost $\boldsymbol{N}^{T} \cdot \boldsymbol{X}^{b}$ of the base case plus the difference between the highest and the lowest dispatch costs under uncertainties. In (11), $\operatorname{Max}_{\boldsymbol{D}^{\max } \in \boldsymbol{\Omega}} \operatorname{Min}_{\boldsymbol{P}}^{u, \max } \boldsymbol{c}^{T} \cdot \boldsymbol{P}^{u, \max }$ and $\operatorname{Min}_{\boldsymbol{D}^{\min } \in \boldsymbol{\Omega}^{M i n}} \operatorname{Pin}^{u, \min } \boldsymbol{c}^{T} \cdot \boldsymbol{P}^{u, \min }$ represent the highest and the lowest dispatch costs under uncertainties, with respect to the base case solution $\widehat{\boldsymbol{X}}^{b}$ and $\widehat{\boldsymbol{P}}^{b}$. $\boldsymbol{D}^{\max } / \boldsymbol{D}^{\min }$ represent realizations of hourly loads and wind outputs that would lead to the highest/lowest dispatch cost.

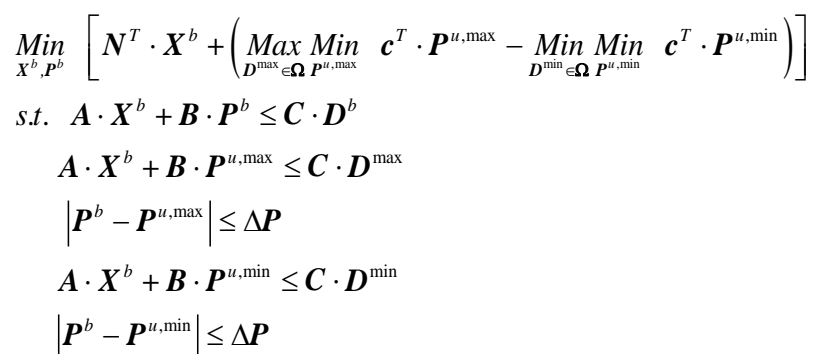

The second/fourth constraint set in (11) includes power balance, power generation limits (restricted by base case UC), and transmission network security constraints for the highest/lowest dispatch cost scenario. The third/fifth constraint set represents that dispatch adjustments of units in response to the highest/lowest dispatch cost scenario are restricted by corrective capacities $\Delta \boldsymbol{P}$ and dispatches in the base case $\boldsymbol{P}^{b}$.

\section{B. MVR SCUC Solution Methodology}

As shown in Fig. 3, the MVR SCUC model is solved via BD as follows:

Step 1: Solve the master UC Problem (12) for the base case, where non-negative ancillary variable $\theta^{\text {max }}$ represents the lower

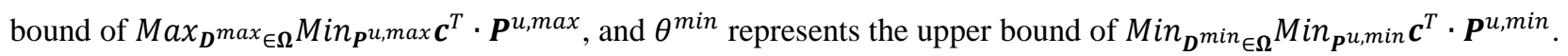

$\underset{\boldsymbol{X}^{b}, \boldsymbol{P}^{b}}{\operatorname{Min}} \boldsymbol{N}^{T} \cdot \boldsymbol{X}^{b}+\theta^{\max }-\theta^{\min }$

s.t. $\boldsymbol{A} \cdot \boldsymbol{X}^{b}+\boldsymbol{B} \cdot \boldsymbol{P}^{b} \leq \boldsymbol{C} \cdot \boldsymbol{D}^{b}$ and all feasibility/optimality Benders cuts

Step 2: Check feasibility by identifying the largest LS. In this step, similar as (3)-(5) in the MLR model, the base case solution $\widehat{\mathbf{P}}^{\mathrm{b}}$ and $\widehat{\mathbf{X}}^{\mathrm{b}}$ from the master problem is checked to identify the largest LS quantity when hourly load and wind generation output vary within the uncertainty set $\boldsymbol{D}$. If the largest LS is higher than the predefined threshold, the feasibility Benders cut (5) will be generated and fed back to the master problem (12), and go back to Step 1. Otherwise, go to Step 3. 
Step 3: Check optimality by calculating the highest dispatch cost variance. If the base case solutions $\widehat{\boldsymbol{P}}^{b}$ and $\widehat{\boldsymbol{X}}^{b}$ pass the feasibility check in Step 2, they will be further evaluated in the optimality check subproblem (13) to identify the highest dispatch cost variance, when hourly load and wind generation output vary within the uncertainty set $\boldsymbol{D}$.

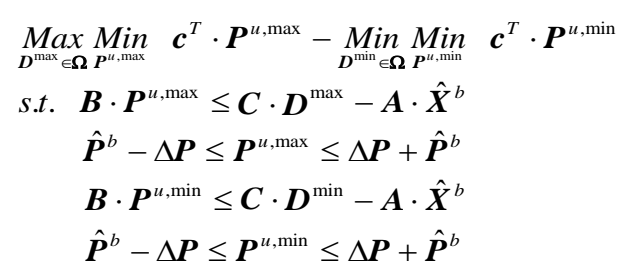

(13) can be divided into two independent subproblems (14) and (15), as $\boldsymbol{P}^{u, \max }$ and $\boldsymbol{P}^{u, \min }$ are naturally decoupled with each other. (14) identifies the realization $\boldsymbol{D}^{\max }$ which leads to the highest dispatch cost, where $\boldsymbol{\lambda}^{\max }, \boldsymbol{\alpha}^{\max }$, and $\boldsymbol{\beta}^{\max }$ are dual variables of corresponding constraints in (14). (14) can be solved by the OA method similar as (8). (15) is used to identify the realization $\boldsymbol{D}^{\mathrm{min}}$ which leads to the lowest dispatch cost, where $\boldsymbol{\lambda}^{\mathrm{min}}, \boldsymbol{\alpha}^{\mathrm{min}}$, and $\boldsymbol{\beta}^{\mathrm{min}}$ are dual variables of corresponding constraints in (15). (15) is an LP problem which can be directly solved.

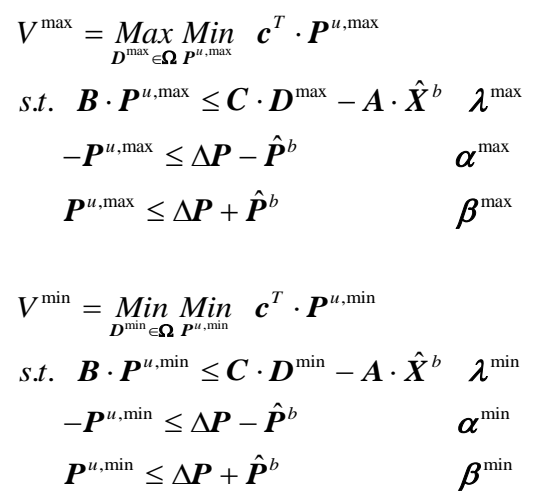

If the objective value $\widehat{V}^{\max }$ in (14) is larger than $\hat{\theta}^{\max }$ obtained from the master problem, the optimality cut (16) will be utilized. Similarly, if the objective value $\widehat{V}^{\text {min }}$ in (15) is smaller than $\hat{\theta}^{\text {min }}$ obtained from the master problem, the optimality cut (17) will be generated. The iterative procedure stops when the master solution satisfies optimality checks. That is, no more optimality cuts are generated in this step.

$$
\begin{aligned}
& \theta^{\max } \geq \hat{V}^{\max }-\left(\boldsymbol{\lambda}^{\max }\right)^{T} \cdot \boldsymbol{A} \cdot\left(\boldsymbol{X}^{b}-\hat{\boldsymbol{X}}^{b}\right)+\left(\boldsymbol{\beta}^{\max }-\boldsymbol{\alpha}^{\max }\right)^{T} \cdot\left(\boldsymbol{P}^{b}-\hat{\boldsymbol{P}}^{b}\right) \\
& \theta^{\min } \leq \hat{V}^{\min }-\left(\boldsymbol{\lambda}^{\min }\right)^{T} \cdot \boldsymbol{A} \cdot\left(\boldsymbol{X}^{b}-\hat{\boldsymbol{X}}^{b}\right)+\left(\boldsymbol{\beta}^{\min }-\boldsymbol{\alpha}^{\min }\right)^{T} \cdot\left(\boldsymbol{P}^{b}-\hat{\boldsymbol{P}}^{b}\right)
\end{aligned}
$$




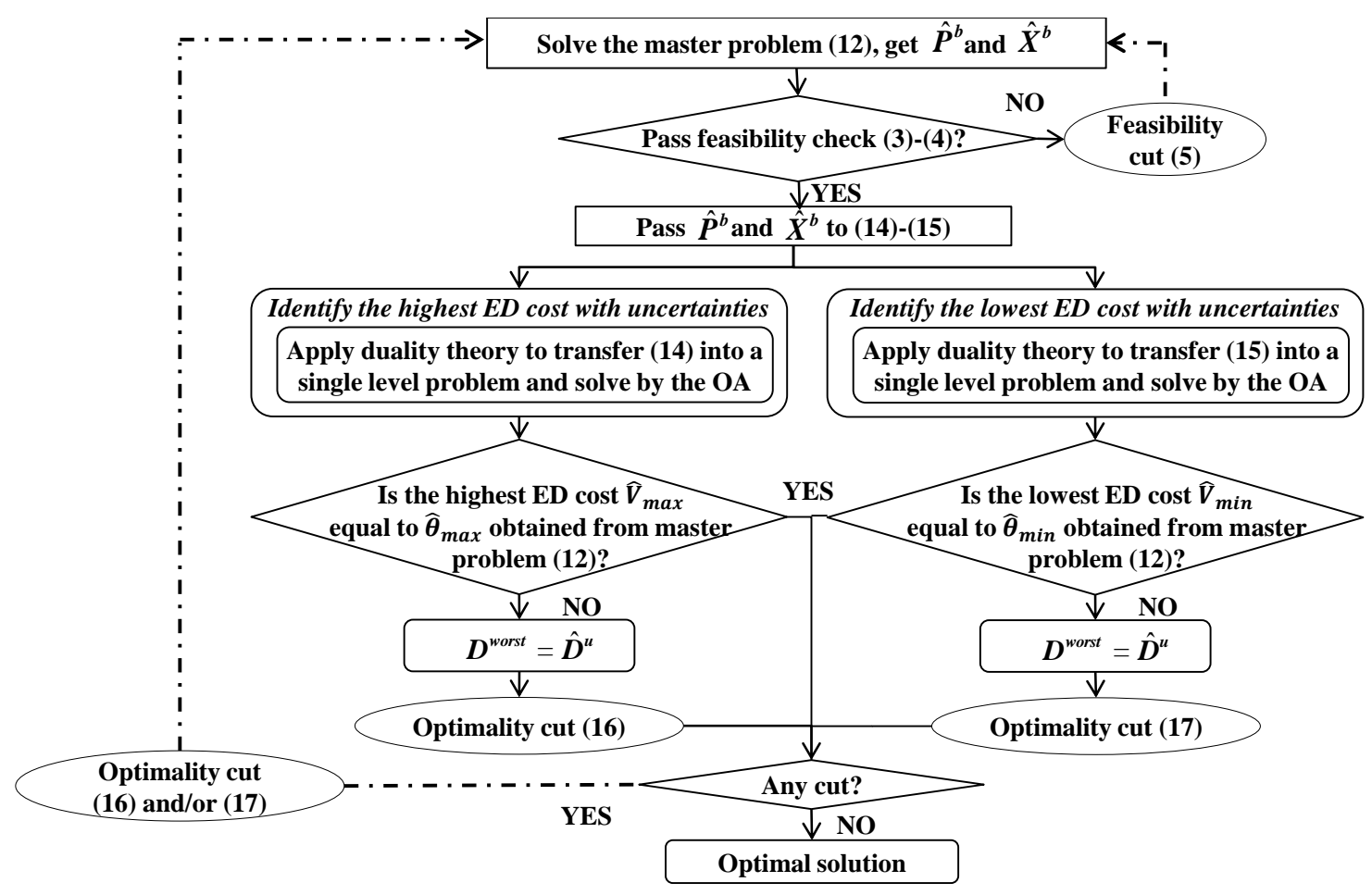

Fig. 3. Solution procedure of the MVR SCUC model

\section{MinimaX REGRET RobUST (MRR) SCUC}

\section{A. MRR SCUC Formulation}

In this paper, regret is defined as the difference between the operation cost of the robust SCUC solution in response to a certain uncertainty realization and that of the SCUC solution by using this uncertainty realization as perfect information. In power systems operation, the ideal situation is that ISOs/RTOs could know the perfect information on real-time uncertainties in the day-ahead timeframe, so that the day-ahead solution would exactly match the real-time situation. Thus, the MRR model adjusts the base case UC solution by minimizing the largest difference between the robust SCUC solution and the SCUC solution with perfect information, and in turn reduces the regret caused by various uncertainties in the real-time operation.

The SCUC solution with perfect information is defined in (18), where $\boldsymbol{X}^{p}$ is a vector of commitment related decisions including on/off and start-up/shut-down statuses of generators with perfect information $\boldsymbol{D}^{u}, \boldsymbol{P}^{p}$ is a vector of dispatch related decisions including generation outputs and power flows with perfect information $\boldsymbol{D}^{u}$, and $\boldsymbol{M}\left(\boldsymbol{D}^{u}\right)$ represents all $\boldsymbol{X}^{p}$ and $\boldsymbol{P}^{p}$ related constraints with respect to the perfect information $\boldsymbol{D}^{u}$.

$\boldsymbol{P e r f e c t}\left(\boldsymbol{D}^{u}\right)=\left\{\underset{\left(\boldsymbol{X}^{p}, \boldsymbol{P}^{p}\right) \in M\left(\boldsymbol{D}^{u}\right)}{\operatorname{Min}} \boldsymbol{N}^{T} \cdot \boldsymbol{X}^{p}+\boldsymbol{c}^{T} \cdot \boldsymbol{P}^{p}\right.$, where $\left.\boldsymbol{M}\left(\boldsymbol{D}^{u}\right)=\left\{\boldsymbol{A} \cdot \boldsymbol{X}^{p}+\boldsymbol{B} \cdot \boldsymbol{P}^{p} \leq \boldsymbol{C} \cdot \boldsymbol{D}^{u}\right\}\right\}$

The MRR SCUC model (19) is to minimize the UC $\operatorname{cost} \boldsymbol{N}^{T} \cdot \boldsymbol{X}^{b}$ of the base case plus the largest regret cost when hourly load and wind power generation are uncertain. 


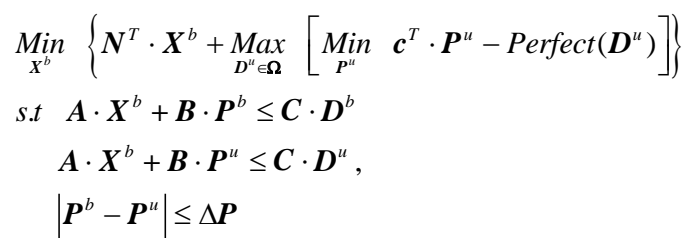

\section{B. MRR SCUC Solution Methodology}

As shown in Fig. 4, the MRR SCUC model is solved via BD as follows:

Step 1: Solve the master UC problem (20) for the base case, where $\theta$ is a non-negative ancillary variable to represent the lower bound of $\operatorname{Max}_{\boldsymbol{D}^{u} \in \boldsymbol{\Omega}}\left[\operatorname{Min}_{\boldsymbol{P}} \boldsymbol{c}^{T} \cdot \boldsymbol{P}^{u}-\operatorname{Perfect}\left(\boldsymbol{D}^{u}\right)\right]$.

$\operatorname{Min}_{X^{b}} \boldsymbol{N}^{T} \cdot \boldsymbol{X}^{b}+\theta$

s.t. $\boldsymbol{A} \cdot \boldsymbol{X}^{b}+\boldsymbol{B} \cdot \boldsymbol{P}^{b} \leq \boldsymbol{C} \cdot \boldsymbol{D}^{b}$ and all feasibility/optimality Benders cuts

Step 2: Check feasibility by identifying the largest LS. In this step, similar as (3)-(5) in the MLR model, the current solution $\widehat{\mathbf{P}}^{\mathrm{b}}$ and $\widehat{\mathbf{X}}^{\mathrm{b}}$ from the master problem is checked to identify the largest LS quantity when hourly load and wind generation vary within the uncertainty set $\mathbf{\Omega}$. If the largest LS quantity is higher than the predefined threshold, the feasibility Benders cut (5) will be generated and fed back to the master problem (20), and go back to Step 1. Otherwise, go to Step 3.

Step 3: Check optimality by calculating the largest regret cost. If base case solutions $\widehat{\boldsymbol{P}}^{b}$ and $\widehat{\boldsymbol{X}}^{b}$ pass the feasibility check in Step 2 , they will be further evaluated in the optimality check subproblem (21) to identify the largest regret cost, when hourly load and wind generation vary within the uncertainty set $\mathbf{\Omega}$.

The second inner objective term in (21) can be transferred into a maximization term by adding an additional negative sign as shown in (22). Thus, by adopting the LP duality theory to transfer the first inner minimization term into a maximization term, (22) can be finally converted into a single-level bilinear optimization problem (23), where $\boldsymbol{\lambda}, \boldsymbol{\alpha}$, and $\boldsymbol{\beta}$ are dual variables of corresponding constraints in (21). However, different from above bilinear problems (3), (8), and (14), (23) is a mixed-integer bilinear problem and the modified outer approximation algorithm [17] is adopted to solve (23).

$$
\begin{aligned}
& V=\underset{D^{u} \in \Omega}{\operatorname{Max}}\left[\underset{\boldsymbol{P}^{u}}{\operatorname{Min}} \boldsymbol{c}^{T} \cdot \boldsymbol{P}^{u}-\boldsymbol{P} \operatorname{erfect}\left(\boldsymbol{D}^{u}\right)\right] \\
& \text { s.t. } \boldsymbol{B} \cdot \boldsymbol{P}^{u} \leq \boldsymbol{C} \cdot \boldsymbol{D}^{u}-\boldsymbol{A} \cdot \hat{\boldsymbol{X}}^{b} \quad \boldsymbol{\lambda} \\
& -\boldsymbol{P}^{u} \leq \Delta \boldsymbol{P}-\hat{\boldsymbol{P}}^{b} \quad \boldsymbol{\alpha} \\
& \boldsymbol{P}^{u} \leq \Delta \boldsymbol{P}+\hat{\boldsymbol{P}}^{b} \quad \boldsymbol{\beta} \\
& V=\underset{\boldsymbol{D}^{u} \in \Omega}{\operatorname{Max}}\left[\underset{\boldsymbol{P}^{u}}{\operatorname{Min}} \boldsymbol{c}^{T} \cdot \boldsymbol{P}^{u}+\underset{\left(\boldsymbol{X}^{p}, \boldsymbol{P}^{p}\right) \in \boldsymbol{M}\left(\boldsymbol{D}^{u}\right)}{\operatorname{Max}}\left(-\boldsymbol{N}^{T} \cdot \boldsymbol{X}^{p}-\boldsymbol{c}^{T} \cdot \boldsymbol{P}^{p}\right)\right]
\end{aligned}
$$




$$
\begin{aligned}
V= & \underset{D^{u} \in \Omega}{\operatorname{Max}}\left(\boldsymbol{C} \cdot \boldsymbol{D}^{u}-\boldsymbol{A} \cdot \boldsymbol{X}^{b}\right)^{T} \cdot \boldsymbol{\lambda}+\left(\Delta \boldsymbol{P}-\hat{\boldsymbol{P}}^{b}\right)^{T} \cdot \boldsymbol{\alpha}+\left(\Delta \boldsymbol{P}+\hat{\boldsymbol{P}}^{b}\right)^{T} \cdot \boldsymbol{\beta}-\boldsymbol{N}^{T} \cdot \boldsymbol{X}^{p}-\boldsymbol{c}^{T} \cdot \boldsymbol{P}^{p} \\
\text { s.t. } & \boldsymbol{B}^{T} \cdot \boldsymbol{\lambda}-\boldsymbol{\alpha}+\boldsymbol{\beta} \leq \boldsymbol{c}, \\
& \boldsymbol{A} \cdot \boldsymbol{X}^{p}+\boldsymbol{B} \cdot \boldsymbol{P}^{p} \leq \boldsymbol{C} \cdot \boldsymbol{D}^{u} \\
& \boldsymbol{\lambda} \leq \mathbf{0}, \quad \boldsymbol{\alpha} \leq \mathbf{0}, \quad \boldsymbol{\beta} \leq \mathbf{0}
\end{aligned}
$$

If the optimal objective $\widehat{V}$ is equal to $\hat{\theta}$ obtained from (20), the current solution $\widehat{\boldsymbol{P}}^{b}$ and $\widehat{\boldsymbol{X}}^{b}$ is the final MRR solution. Otherwise, the optimality cut (24) will be generated and added to the next iteration of the master problem for seeking robust UC solutions that would minimize the worst case regret cost. The iterative procedure stops when the master solution satisfies optimality checks and no more optimality cuts are generated.

$$
\theta \geq \hat{V}-\lambda^{T} \cdot \boldsymbol{A} \cdot\left(\boldsymbol{X}^{b}-\hat{\boldsymbol{X}}^{b}\right)+(\boldsymbol{\beta}-\boldsymbol{\alpha})^{T} \cdot\left(\boldsymbol{P}^{b}-\hat{\boldsymbol{P}}^{b}\right)
$$

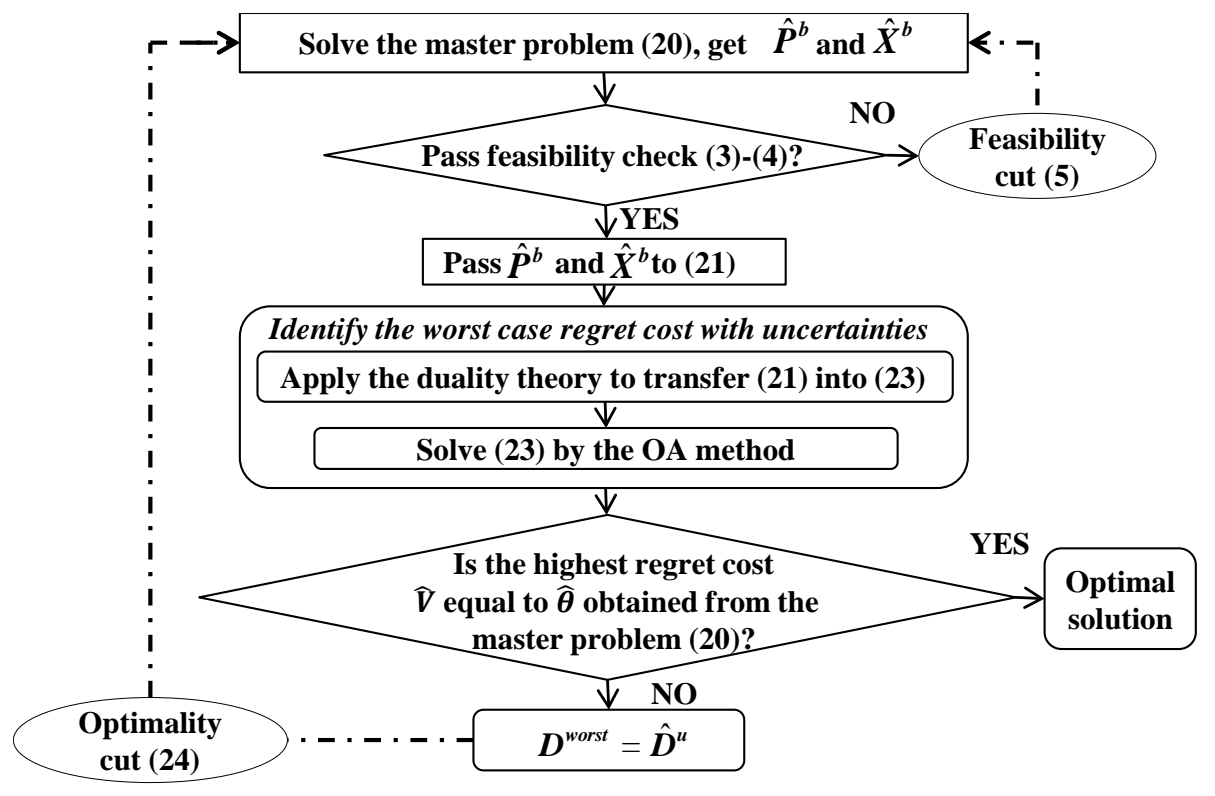

Fig. 4. Solution procedure of the MRR SCUC model

\section{NUMERICAL CASE STUdiES}

The modified IEEE 118-bus system is used to analyze the performance of the four robust SCUC models and their impacts on the operational security and economics of power systems under uncertainty. The system includes 54 thermal units, 3 wind farms, 186 lines, and 91 loads [2]. The 24-hour wind power generation and load profiles are modified based on actual data from the NYISO market [19], as shown in Fig. 5. Load and wind uncertain intervals are set based on day-ahead forecast values $P_{d t}^{b}$ and $P_{f, w t}^{b}$, together with their variations $\quad 0.1 \cdot P_{d t}^{b} \quad$ and $\quad 0.2 \cdot P_{f, w t}^{b} \quad[19]$. That $\quad$ is, $\quad\left[P_{d t}^{b}-0.1 \cdot P_{d t}^{b}, P_{d t}^{b}+0.1 \cdot P_{d t}^{b}\right] \quad$ and $\left[P_{f, w t}^{b}-0.2 \cdot P_{f, w t}^{b}, P_{f, w t}^{b}+0.2 \cdot P_{f, w t}^{b}\right]$. 


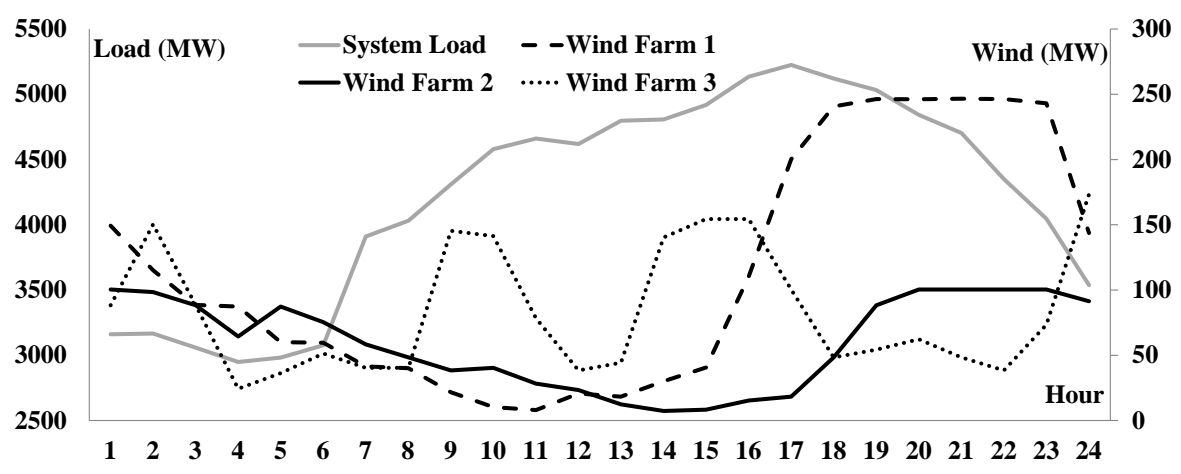

Fig. 5. System load profile and wind power generation of the three wind farms

In numerical case studies, wind uncertainty budget level $\Delta_{w}$ is fixed as $N T$, which means wind uncertainty shows a $20 \%$ deviation from the day-ahead forecasted value at each hour $t$. Three load uncertainty budget levels, $0, N T / 2$, and $N T$, are explored. $\Delta_{d}$ of zero means $0 \%$ load uncertainty, and the robust SCUC solution only addresses uncertain wind generation. When $\Delta_{d}$ is $N T$, the entire load uncertain interval $\left[P_{d t}^{b}-0.1 \cdot P_{d t}^{b}, P_{d t}^{b}+0.1 \cdot P_{d t}^{b}\right]$ is covered. LS threshold for uncertainty evaluation subproblems is set as $10^{-3} \mathrm{MWh}$. MILP gap is set as $10^{-5}$.

\section{A). Base Case Cost of Robust Solutions from Different Models}

Table 1 shows results of the base case $\mathrm{UC} \operatorname{cost} \mathrm{N}^{\mathrm{T}} \cdot \mathrm{X}^{\mathrm{b}}$, the base case dispatch cost $\mathrm{c}^{\mathrm{T}} \cdot \mathrm{P}^{\mathrm{b}}$, and the total operation cost of the base case $\mathrm{N}^{\mathrm{T}} \cdot \mathrm{X}^{\mathrm{b}}+\mathrm{c}^{\mathrm{T}} \cdot \mathrm{P}^{\mathrm{b}}$ with respect to robust SCUC solutions from the four models. Figs. 6-8 show the number of committed units for the four robust SCUC models with the budget level $\Delta_{\mathrm{d}} / \mathrm{NT}$ of $0.0,0.5$, and 1.0, respectively. In Figs. 6-8, the x-axis represents the time horizon, and the y-axis shows the number of committed units for each robust SCUC model at each hour.

Table 1 shows that, for each robust SCUC model, with a larger uncertainty budget level $\Delta_{\mathrm{d}} / \mathrm{NT}$, UC costs, dispatch costs, and total operation costs are all monotonously increasing. In turn, more units are committed in the base case to cover a larger uncertainty range with the increased uncertainty budget level $\Delta_{\mathrm{d}} / \mathrm{NT}$, as shown in Figs. 6-8. Table 1 also shows that MER has the lowest UC cost, which means the smallest number of units committed in the base case, while MLR has the highest UC cost with the largest number of online units as shown in Figs. 6-8. For instance, with $\Delta_{\mathrm{d}} / \mathrm{NT}$ of 1.0, 50 units are committed at peak hour 17 in MLR, while only 44 units are committed in MER, 45 units are committed in MVR and MRR as shown in Fig. 8. On the other hand, MLR has the lowest dispatch cost and MRR has the highest base case dispatch cost. With $\Delta_{\mathrm{d}} / \mathrm{NT}$ of 1.0 , the lowest UC cost is $\$ 1,264.950 .45$ from MER, the second lowest UC cost is $\$ 1,268,609.52$ from MVR, and the highest UC costs is $\$ 1,358,596.65$ from MLR. Moreover, the ascending orders of dispatch costs are $\$ 1,025,721.42, \$ 1,270,940.27, \$ 1,424,285.80$, and $\$ 1,451,526.58$ for MLR, MER, MVR, and MRR, respectively. The main reason is that, the objective of the MLR model is to minimize the total operation cost of the base case, while objectives of the other three robust models are to minimize the base case UC cost plus additional cost components with respect to different worst case definitions. Thus, in recognizing that a higher UC cost (i.e., startup 
cost and no-load cost) is usually accompanied with a lower dispatch cost for a generator, the MLR model, by balancing UC cost and dispatch cost to minimize the total operation cost, tends to present higher UC cost but lower dispatch cost as compared to the other three models.

Table 1 Base Case Costs of Different Models (\$)

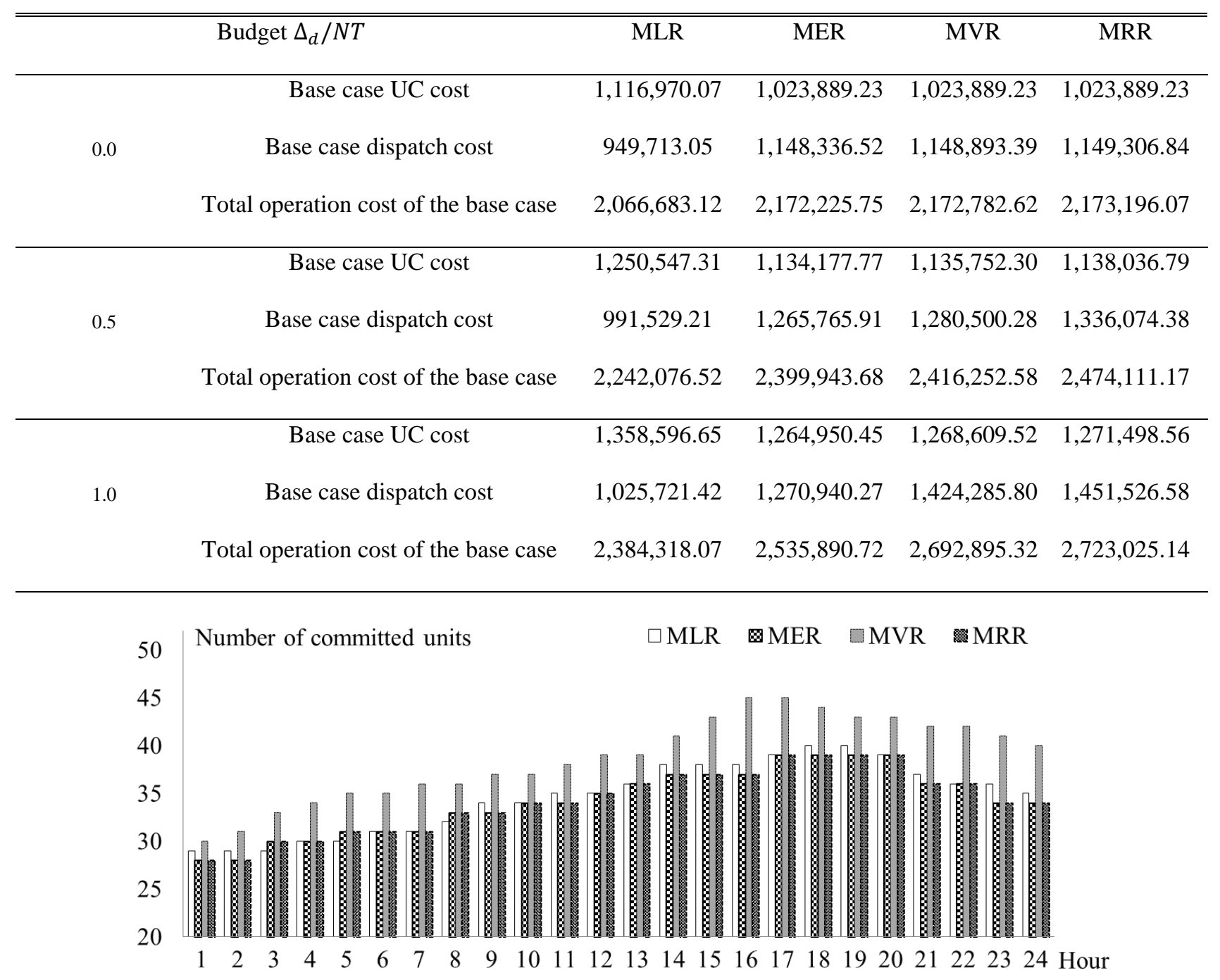

Fig. 6. The number of committed units of the four robust SCUC models with the uncertainty budget level of 0.0

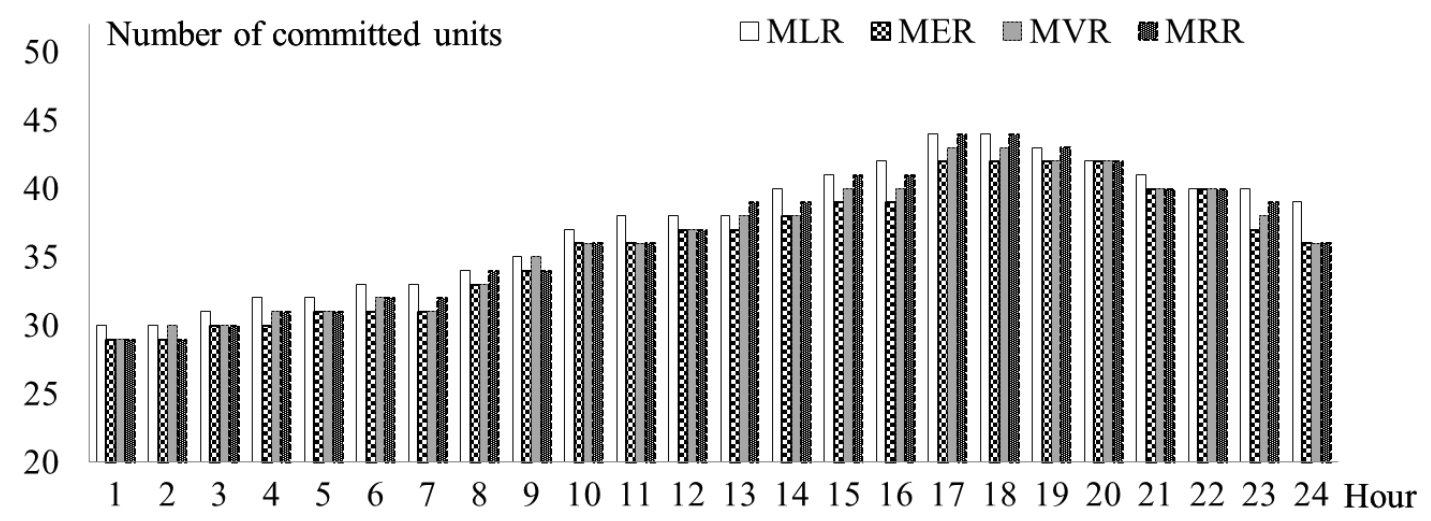

Fig. 7. The number of committed units of the four robust SCUC models with the uncertainty budget level of 0.5 


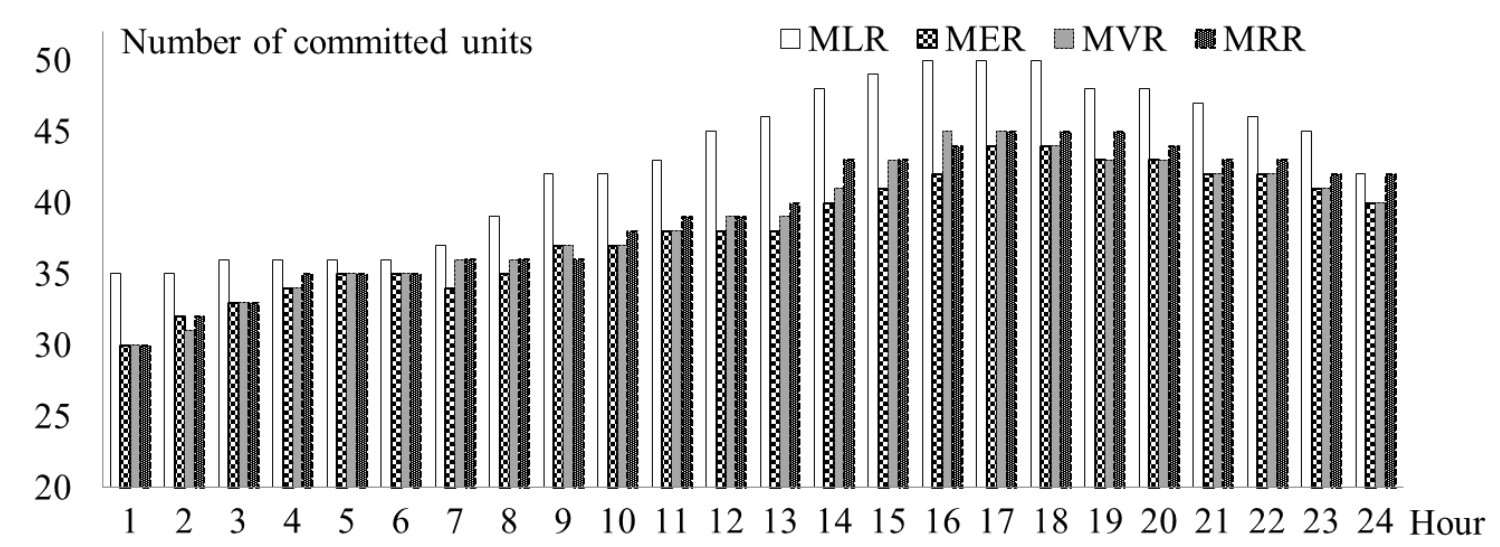

Fig. 8. The number of committed units of the four robust SCUC models with the uncertainty budget level of 1.0

All cases studies utilize CPLEX 12.4.0.0 on an Intel Core i7 3.40-GHz personal computer. The MLR model takes 7 feasibility check iterations in about 804 seconds to reach the final optimal solution. The MER model is solved with 11 feasibility check iterations and 3 optimality check iterations in about 1027 seconds. The MVR model takes 11 feasibility check iterations and 4 optimality check iterations to derive the final optimal solution in about 1034 seconds, and MRR takes 11 feasibility check iterations and 3 optimality check iterations in about 1029 seconds. Since MER, MVR, and MRR models need both feasibility and optimality checks, their computational times are higher than that of MLR. In addition, as the master problem of the MLR model, by simultaneously minimizing the base case UC cost and dispatch cost, tends to commit more units in the base case, fewer iterations are needed to pass the feasibility check than the other three models.

\section{B). Robustness Tests of Robust Solutions}

This section shows that because of different worst case definitions, the four models present variant robust test performances. Latin Hypercube Sampling (LHS) [2] is adopted to simulate uncertainties via 10000 scenarios, for testing the performance of the four robust models. Load and wind generation uncertainties are assumed to follow normal distributions $N\left(P_{d t}^{b},\left(\sigma_{d t}^{b}\right)^{2}\right)$ and $N\left(P_{f, w t}^{b},\left(\sigma_{f, w t}^{b}\right)^{2}\right)$. As the uncertainty sets $\left[P_{d t}^{b}-0.1 \cdot P_{d t}^{b}, P_{d t}^{b}+0.1 \cdot P_{d t}^{b}\right]$ and $\left[P_{f, w t}^{b}-0.2 \cdot P_{f, w t}^{b}, P_{f, w t}^{b}+0.2 \cdot P_{f, w t}^{b}\right]$ could cover about 85\% of NYISO historical load and wind realizations in Jun-Aug 2013 [18], the same 85\% coverage levels are applied to determine parameters $\sigma_{d t}^{b}$ and $\sigma_{f, w t}^{b}$. That is, $z_{0.85} \cdot \sigma_{d t}^{b}=0.1 \cdot P_{d t}^{b}$ and $z_{0.85} \cdot \sigma_{f, w t}^{b}=0.2 \cdot P_{f, w t}^{b}$, where $z_{0.85}=$ 1.4395 represents the $85 \%$ confidence level of the standard normal distribution. Therefore, $P_{d t}^{u}$ follows $N\left(P_{d t}^{b},\left(0.1 \cdot P_{d t}^{b} / 1.4395\right)^{2}\right)$ and $P_{f, w t}^{u}$ follows $N\left(P_{f, w t}^{b},\left(0.2 \cdot P_{f, w t}^{b} / 1.4395\right)^{2}\right)$.

\section{B.1). LS quantification}

LS results of the four robust SCUC models are shown in Table 2, which include average LS quantities and the largest LS 
quantities over 10000 scenarios with respect to robust SCUC solutions in Table 1. The LS test is done by solving the redispatch problem for each scenario, which is restricted by UC and dispatch solutions $\widehat{\boldsymbol{X}}^{b}$ and $\widehat{\boldsymbol{P}}^{b}$ in the base case as well as corrective capacity limits. By allowing load shedding for each load bus, no other system constraints will be violated.

Table 2 Average and The largest LS (MWh)

\begin{tabular}{cccccc}
\hline \hline \multicolumn{2}{c}{ Budget $\Delta_{d} / N T$} & MLR & MER & MVR & MRR \\
\hline \multirow{2}{*}{0.0} & Average LS & 58.01 & 58.26 & 59.23 & 58.93 \\
\cline { 2 - 6 } & The largest LS & 485.57 & 486.18 & 498.39 & 486.26 \\
\multirow{2}{*}{0.5} & Average LS & 25.01 & 26.50 & 26.89 & 26.10 \\
& The largest LS & 277.29 & 271.94 & 280.75 & 276.43 \\
\multirow{2}{*}{1.0} & Average LS & 0.004 & 0.004 & 0.003 & 0.004 \\
& The largest LS & 18.54 & 18.61 & 19.04 & 18.67 \\
\hline
\end{tabular}

Table 2 shows that with a higher budget level $\Delta_{d} / N T$, both average and the largest LS in each column are monotonously decreasing, since the system has more corrective capacities with more committed units. For instance, average LS values are 58.26MWh and $0.004 \mathrm{MWh}$, and the largest LS values are $486.18 \mathrm{MWh}$ and $18.61 \mathrm{MWh}$ for the MER model, when $\Delta_{d} / N T$ is 0.0 and 1.0, respectively. In addition, with the same uncertainty budget, LS quantities of the four robust models are similar. That is, the four models guarantee similar security levels of power systems operation under uncertainty in terms of LS quantities.

It is also noted that LS still exists even when the entire uncertain range is considered (i.e., $\Delta_{d} / N T$ is 1.0). For instance, the largest LS values are 18.54MWh, 18.61MWh, 19.04MWh, and 18.67MWh for the four robust SCUC models. The reason is that when using normal distributions, out-of-sample scenarios beyond the predefined uncertainty sets $\left[P_{d t}^{b}-0.1 \cdot P_{d t}^{b}, P_{d t}^{b}+0.1 \cdot P_{d t}^{b}\right]$ and $\left[P_{f, w t}^{b}-0.2 \cdot P_{f, w t}^{b}, P_{f, w t}^{b}+0.2 \cdot P_{f, w t}^{b}\right]$ may not be fully handled by the robust SCUC solutions. However, the number of these scenarios would be limited and in turn the resulting average LS quantity would be small.

\section{B.2). Economic dispatch cost quantification}

ED results of the 10000 scenarios with respect to the four robust SCUC solutions in Table 1 are shown in Table 3. Scenario ED solutions are calculated by solving the redispatch problem for each scenario, which is restricted by $\widehat{\boldsymbol{X}}^{b}$ and $\widehat{\boldsymbol{P}}^{b}$ in the base case as well as corrective capacity limits. The penalty cost for LS is set as $\$ 1000 / \mathrm{MWh}$.

Table 3 reports the highest dispatch cost and the difference between the highest and the lowest dispatch costs over 10000 scenarios. It shows that with a wider range of load uncertainties, the highest dispatch cost and the difference between the highest and the lowest dispatch costs in each column are monotonously decreasing. For instance, the highest dispatch costs are 
$\$ 2,138,229.62$ and $\$ 1,876,472.69$ for MLR when $\Delta_{d} / N T$ are 0.0 and 1.0 , respectively, and the corresponding dispatch cost differences are $\$ 940,593.37$ and $\$ 641,648.70$. The reason is that fewer units are committed with a smaller uncertainty range. Thus, larger LS could happen when solving the redispatch problem for certain scenarios, which could result in higher LS penalty cost and in turn higher dispatch cost difference.

Table 3 The Largest ED cost and The largest ED cost difference (\$)

\begin{tabular}{|c|c|c|c|c|c|}
\hline & Budget $\Delta_{d} / N T$ & MLR & MER & MVR & MRR \\
\hline \multirow{2}{*}{0.0} & The highest dispatch cost & $2,138,229.62$ & $2,055,854.93$ & $2,107,368.54$ & $2,086,026.77$ \\
\hline & Difference between the highest/lowest dispatch & $940,593.37$ & $685,379.98$ & $670,926.76$ & $674,296.25$ \\
\hline \multirow{2}{*}{0.5} & The highest dispatch cost & $1,966,212.75$ & $1,919,484.24$ & $1,931,837.06$ & $1,962,439.63$ \\
\hline & Difference between the highest/lowest dispatch & $940,288.98$ & $652,730.76$ & $639,701,84$ & $645,785.11$ \\
\hline \multirow{2}{*}{1.0} & The highest dispatch cost & $1,876,472.69$ & $1,812,312.10$ & $1,830,821.62$ & $1,858,000.75$ \\
\hline & Difference between the highest/lowest dispatch & $641,648.70$ & $542,220.56$ & $438,177.58$ & $447,335.34$ \\
\hline
\end{tabular}

It is also noticed that MVR shows the best performance in handling real-time uncertainties in terms of the smallest dispatch cost difference. For instance, the difference between the highest and the lowest dispatch costs over 10000 scenarios is $\$ 438,177.58$ for the MVR model with $\Delta_{d} / N T$ of 1.0 , which is smaller than those of the other three models. This is well expected because the MVR model is formulated to minimize the UC cost of the base case plus the difference between the highest and the lowest dispatch costs under uncertainties.

In addition, the MER model shows the best performance in handling the real-time dispatch cost in terms of the smallest value of the highest dispatch cost. For instance, the highest dispatch cost over 10000 scenarios is $\$ 1,812,312.10$ for the MER model with $\Delta_{d} / N T$ of 1.0, which is smaller than those of the other three models. This is also well expected because the MER model intends to minimize the UC cost of the base case plus the highest dispatch cost under uncertainties.

\section{B.3). Regret cost quantification}

Table 4 shows the regret cost performance of the four robust SCUC solutions in Table 1. The regret cost is calculated by measuring the difference between the operation cost by solving the redispatch problem for each scenario with respect to the robust SCUC solution and that of the SCUC problem by using this scenario as perfect information.

Table 4 shows the highest regret values over 10000 scenarios. It is noted that with a larger budget $\Delta_{d} / N T$ setting, the highest regret values in each column are monotonously decreasing. For instance, the highest regret values are $\$ 897,150.18$ and $\$ 361,004.11$ for the MLR model when $\Delta_{d} / N T$ is 0.0 and 1.0, respectively. The reason is that, with a smaller uncertainty range, 
fewer units are committed and higher LS could happen in certain uncertainty realizations. Thus, a higher penalty cost would occur, which in turn increases the total operation cost and enlarges the regret value.

Table 4 Highest Regret Cost of Different Models (\$)

\begin{tabular}{ccccc}
\hline Budget $\Delta_{d} / N T$ & MLR & MER & MVR & MRR \\
\hline 0.0 & $897,150.18$ & $891,993.06$ & $889,360.56$ & $882,869.61$ \\
0.5 & $448,815.41$ & $394,122.98$ & $378,672.03$ & $365,414.16$ \\
1.0 & $361,004.11$ & $294,781.40$ & $286,067.69$ & $248,804.43$
\end{tabular}

In addition, since the MRR model intends to minimize the base case UC cost plus the maximum regret cost when hourly load and wind generation are uncertain, Table 4 shows that the MRR model has the best performance in terms of the smallest value of the maximum regret cost. On the other hand, the MLR model presents much higher regret values than the other three models. For instance, the largest regret value of MLR is $\$ 361,004.11$ with $\Delta_{d} / N T$ of 1.0 , which is much higher than $\$ 294,781.40, \$ 286,067.69$, and $\$ 248,804.43$ of the other three models. From the data in Tables 1 and 4 , we can see that the robust solution of the MLR model would be more conservative than the other three. As shown in Table 1, the robust solution of MLR commits more units in the base case. However, in observing individual scenarios with perfect information, we may not need to commit so many units to handle individual realizations, which in turn causes the regret value of the MLR SCUC solution to be the highest.

\section{B.4). Locational marginal price (LMP) quantification}

LMPs from the four robust SCUC models are also analyzed. Table 5 shows LMPs on buses 10 and 93 at hours 2 and 17 with $\Delta_{d} / N T=1.0$. Bus 10 is a generation bus while bus 93 is a load bus. For each model, the top data represents the base case LMP, and the bottom one represents the average and the standard deviation of LMPs over 10000 scenarios.

Since the four models present different UC and ED solutions in the base case, their base case LMPs are different. On the other hand, off-peak LMPs are usually expected to be lower than those of peaks in a certain location, especially in smart grid that demand response is promoted. For instance, at the load bus 93, peak LMPs in the base case from the four models are all higher than those of off-peaks. In addition, it is also noticed that LMPs from MER, MVR, and MRR tend to be higher than MLR, which is driven by the reason that MLR commits more generators with lower dispatch costs.

Table 5 LMPs of the Four Models with $\Delta_{d} / N T=1.0(\$ / \mathrm{MWh})$

\begin{tabular}{cccccc}
\hline \hline Hour & Bus & MLR & MER & MVR & MRR \\
\hline \multirow{2}{*}{2} & 1 & 22.98 & 37.66 & 30.42 & 37.90 \\
& 0 & $22.98 \pm 0.00$ & $30.09 \pm 13.46$ & $37.71 \pm 13.04$ & $35.80 \pm 12.24$
\end{tabular}




\begin{tabular}{|c|c|c|c|c|c|}
\hline \multirow{2}{*}{2} & 9 & 37.61 & 37.66 & 48.19 & 50.87 \\
\hline & 3 & $38.25 \pm 2.14$ & $33.57 \pm 24.04$ & $48.08 \pm 20.13$ & $49.65 \pm 27.06$ \\
\hline \multirow{2}{*}{17} & 1 & 28.13 & 39.16 & 30.71 & 37.55 \\
\hline & 0 & $28.92 \pm 6.25$ & $54.65 \pm 15.27$ & $90.69 \pm 19.07$ & $47.39 \pm 14.61$ \\
\hline \multirow{2}{*}{17} & 9 & 72.45 & 77.28 & 98.61 & 90.78 \\
\hline & 3 & $79.69 \pm 27.82$ & $67.29 \pm 38.49$ & $120.04 \pm 210.45$ & $89.46 \pm 84.37$ \\
\hline
\end{tabular}

Table 5 also shows that scenario LMPs from the four models presents difference levels of volatility, in terms of standard deviations of LMPs over 10000 scenarios. At both generation and load buses, standard deviations of scenario LMPs from the MLR model are all smaller than those of the other three models. Especially, at off-peak hour 2, scenario LMPs on bus 10 are all equal to 22.98 \$MWh with zero standard deviation. In addition, at peak hour 17, scenario LMPs on buses 10 and 93 in the MLR model are also less volatile than the other three models (i.e., the standard deviations of $6.25 \$ / \mathrm{MWh}$ and $27.82 \$ / \mathrm{MWh}$ are smaller than the others). The most significant volatility of $210.45 \$ / M W h$ is seen from the MVR model on bus 93 at hour 17 . When LS happens on a bus, scenario LMPs will be impacted by the penalty cost of LS (i.e., $\$ 1000 / \mathrm{MWh}$ ). Since the MLR model commits more generators with lower dispatch costs, LS occurs only in a smaller number of scenarios as compared to the other three models, and in turn its scenario LMPs are less volatile. Since scenario LMPs represent possible real-time LMP values when a certain scenario comes into reality, a larger standard deviation of scenario LMPs or a larger gap between the base case LMP and the average LMP may introduce more volatility into the real-time market.

\section{C). Do-Not-Exceed (DNE) Limit Quantification}

This case study explores the largest potential uncertainty handling capacity of the four robust models, by determining the maximum load uncertainty ranges that each robust solution can accommodate without sacrificing system security. The largest potential uncertainty handling capacity is quantified by the do-not-exceed (DNE) limit [20], which is calculated based on the robust UC and dispatch solutions $\boldsymbol{X}^{b}$ and $\boldsymbol{P}^{b}$ in the base case via (25).

$$
\begin{aligned}
\operatorname{Max} & \left(\boldsymbol{D}^{+}+\boldsymbol{D}^{-}\right)^{T} \cdot \boldsymbol{D}^{b} \\
\text { s.t } & \boldsymbol{B} \cdot \boldsymbol{P}^{u} \leq \boldsymbol{C} \cdot \boldsymbol{D}^{u}-\boldsymbol{A} \cdot \hat{\boldsymbol{X}}^{b} \\
& \hat{\boldsymbol{P}}^{b}-\Delta \boldsymbol{P} \leq \boldsymbol{P}^{u} \leq \hat{\boldsymbol{P}}^{b}+\Delta \boldsymbol{P} \\
& D_{d t}^{b} \cdot\left(1-D_{d t}^{-}\right) \leq D_{d t}^{u} \leq D_{d t}^{b} \cdot\left(1+D_{d t}^{+}\right) \quad \forall d, t \\
& \boldsymbol{D}^{+} \geq 0, \boldsymbol{D}^{-} \geq 0
\end{aligned}
$$

The objective of (25) is to find the largest operation range that a robust SCUC solution can accommodate, i.e., the system remains feasible under any realization within the range. Uncertain dispatch $\boldsymbol{P}^{u}$ is restricted by the base case solutions $\boldsymbol{X}^{b}$ and $\boldsymbol{P}^{b}$ as well as corrective constraints. $\boldsymbol{D}^{b} \cdot\left(1+\boldsymbol{D}^{+}\right)$and $\boldsymbol{D}^{b} \cdot\left(1-\boldsymbol{D}^{-}\right)$represent upper and lower bounds of the DNE limits of $\boldsymbol{d}$. Robust 
solutions of the four models with $\Delta_{d} / N T$ of 1.0 are used to conduct the DNE test. Fig. 9 shows DNE limits of system load. It indicates that system load DNE limits from the four models all cover the predefined deterministic uncertainty interval $\left[P_{d t}^{b}-0.1 \cdot P_{d t}^{b}, P_{d t}^{b}+0.1 \cdot P_{d t}^{b}\right]$. It also shows that percentage DNE limits at off-peak hours are higher than those at peak hours. For instance, at hour 1, upper bounds of the percentage DNE limit $\boldsymbol{D}^{+}$are 13.57\%, 13.07\%, 13.48\%, and 13.23\% for MLR, MER, MVR, and MRR, respectively, and the corresponding lower bounds $\boldsymbol{D}^{-}$are $15.54 \%, 14.07 \%, 13.98 \%$, and $13.73 \%$. On the other hand, at peak hour 17 , the percentage DNE limit upper bounds $\boldsymbol{D}^{+}$are $11.61 \%, 10.47 \%, 10.15 \%$, and $10.03 \%$, and the lower bounds $\boldsymbol{D}^{-}$are $12.10 \%, 10.97 \%, 10.64 \%$, and $10.53 \%$. Although $\boldsymbol{D}^{+}$and $\boldsymbol{D}^{-}$at off-peak hours are larger, the actual DNE MW limits at peak hours are higher than those of off-peaks because $\boldsymbol{D}^{b}$ in peak hours are much higher. For instance, at hour 1, upper bounds of the DNE limit $\boldsymbol{D}^{b} \cdot \boldsymbol{D}^{+}$are 533.62MW, 514.17MW, 530.12MW, and 520.24 MW for MLR, MER, MVR, and MRR, respectively, and the corresponding lower bounds $\boldsymbol{D}^{b} \cdot \boldsymbol{D}^{-}$are $611.10 \mathrm{MW}, 553.50 \mathrm{MW}, 549.78 \mathrm{MW}$, and 539.91 MW. On the other hand, at peak hour 17, the DNE limit upper bounds are 666.95 MW, 601.68 MW, 583.05 MW, and 576.27 MW, and the lower bounds are 695.69 MW, 630.41 MW, 611.79 MW, and 605.01 MW. This indicates that in order to have the equal percentage DNE limit performance over different hours, or even higher percentage DNE limit performance at peak hours (since usually peak hours is most concerned because of congestions and resource limitations), it would be better to define uncertainty interval $P_{d t}^{u} \in$ $\left[P_{d t}^{b}-\tilde{P}_{d t}, P_{d t}^{b}+\tilde{P}_{d t}\right]$ using different percentages of $P_{d t}^{b}$ at different hours.

Fig. 9 also shows that the MLR model derives wider DNE ranges than the other three models, and in turn could accommodate more uncertainties without sacrificing system security. The main reason is that, as compared to the other three models, the MLR model commits more units in the base case at the cost of a higher base case UC cost, as shown in Table 1. Thus, it could provide more corrective capabilities and present larger potential abilities to accommodate more uncertainties.

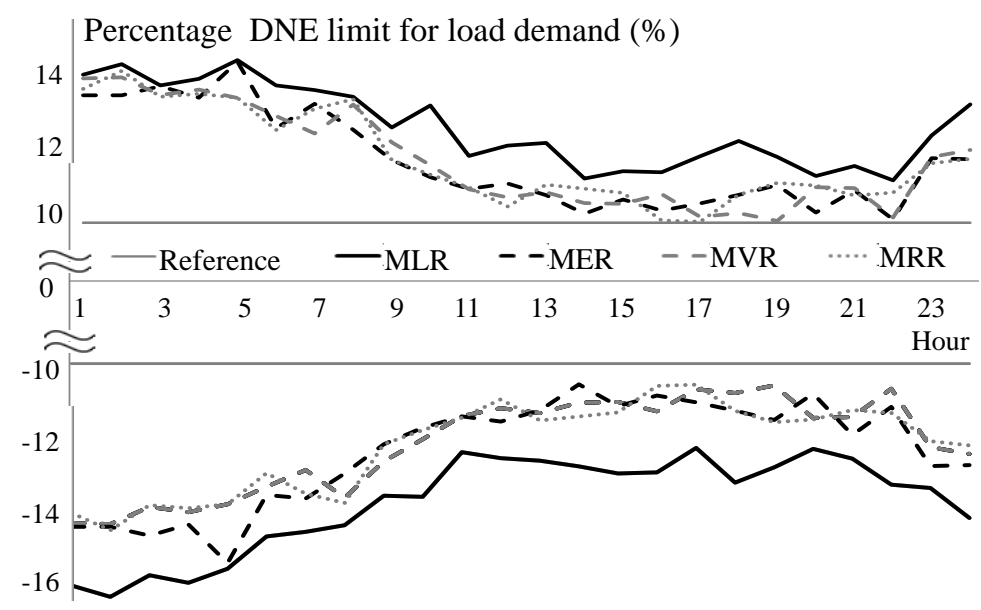

Fig. 9. System load DNE limits from the four models

Table 6 summarizes the performance of the four robust models in terms of base case UC cost, base case dispatch cost, LS, 
scenario dispatch cost, LMP, regret cost, and DNE limits. It shows that MLR presents a better performance in terms of base case dispatch cost, LMPs, and DNE limit. MER works better in terms of base case UC cost and the highest dispatch cost over scenarios. On the other hand, MVR performs better in terms of the difference between the highest and the lowest dispatch costs over scenarios, which means a lower variance of operation cost when dealing with real-time uncertainties, and MRR presents the lowest regret cost. In addition, the four models present similar security levels of power systems operation under uncertainty in terms of LS quantities.

Table 6 Performance Summary of the Four Robust SCUC Models (1/4 Means the Best/Worst Performance)

\begin{tabular}{ccccc}
\hline \hline & MLR & MER & MVR & MRR \\
\hline Base case UC cost & 4 & 1 & 2 & 3 \\
Base case dispatch cost & 1 & 2 & 3 & 4 \\
LS & 1 & 1 & 1 & 1 \\
Highest scenario dispatch cost & 4 & 1 & 2 & 3 \\
Scenario dispatch cost difference & 4 & 3 & 1 & 2 \\
LMP & 1 & 2 & 4 & 3 \\
Regret cost & 4 & 3 & 2 & 1 \\
DNE limit & 1 & 2 & 3 & 4 \\
\hline
\end{tabular}

\section{CONCLUSION}

This paper investigates four robust SCUC models based on different worst case definitions. BD and OA are used to solve the four robust models. Numerical tests on the modified IEEE 118-bus system show the performance of the four robust models. The MLR model with the uncertainty budget limit of 0.0 corresponds to the deterministic SCUC practice in the industry. In comparison, although all the four models can adaptively handle any uncertainty realizations within the predefined uncertainty set, they show different levels of advantages over base case costs, scenario dispatch costs, regret costs, DNE limits, and LMPs.

Market economic operation signals (such as LMPs, regret costs, and base case costs) and system secure operation signals (such as LS quantities and DNE limits) align the financial interest of market participants with both system and market operation objectives, and reflect system dynamical operation conditions related to system physical constraints. Thus, by responding to effective base case costs, scenario dispatch costs, regret costs, DNE limits, and LMPs signals, market participants could help address system operation needs and achieve economic efficiency. In this paper, numerical tests show that all the four robust models outperform the deterministic SCUC model (i.e., the MLR model with the uncertainty budget limit of 0.0) in terms of better economic and secure operation. The four models present similar security levels of power systems operation under uncertainty in 
terms of LS quantities. In addition, the MLR model derives wider DNE ranges than the other three models, and in turn could accommodate more uncertainties without sacrificing system security. On the other hand, the MLR model presents a better LMP performance in terms of lower off-peak LMPs in the base case and smaller standard LMP deviations under uncertainties, as well as lower dispatch costs and wider DNE limits. MER has a better performance in terms of base case UC cost and the highest dispatch cost over scenarios. In terms of the difference between the highest and the lowest dispatch costs over scenarios, which indicates the variance of operation costs when dealing with real-time uncertainties, MVR will be the first choose for the system operators. Finally, MRR performs better in terms of the regret cost which indicates the difference between the operation cost by solving the redispatch problem for each scenario and that of the SCUC problem by using this scenario as perfect information.

Future research work could investigate approaches to leverage the four robust models and derive effective LMP signals for the market clearing under the robust SCUC framework, which would incentivize market participants to effectively follow ISOs/RTOs' dispatch instructions for ensuring system security and market economics.

\section{ACKNOWLEDGMENT}

This study is funded in part by the U.S. National Science Foundation grant ECCS-1254310 and U.S. Department of Energy Grants \# DE-FOA-0000152.

\section{REFERENCES}

[1] M. Shahidehpour, H. Yamin, and Z. Li, Market Operations in Electric Power System. New York, NY, USA: Wiley, 2002.

[2] L. Wu, M. Shahidehpour, and T. Li, "Stochastic security-constrained unit commitment," IEEE Trans. Power Syst., vol. 22, no. 2, pp. 800-811, May 2007.

[3] H. Wu, M. Shahidehpour, Z. Li, and W. Tian, "Chance-constrained day-ahead scheduling in stochastic power system operation," IEEE Trans. Power Syst., vol. 29, no. 4, pp. 1583-1591, Jul. 2014.

[4] D. Bertsimas, E. Litvinov, X.A, Sun, J. Zhao, and T. Zheng, “Adaptive robust optimization for the security constrained unit commitment problem," IEEE Trans. Power Syst., vol. 28, no. 1, pp. 52-63, Feb. 2013.

[5] G. Yongpei and W. Jianhui, "Uncertainty sets for robust unit commitment," IEEE Trans. Power Syst., vol. 29, no. 3, pp. 1439-1440, May 2014.

[6] B. Hu, L. Wu, and M. Marwali, "On the robust solution to SCUC with load and wind uncertainty correlations," IEEE Trans. Power Syst., vol. 29, no. 6, pp. 2952-2964, Nov. 2014.

[7] R. Jiang, J. Wang, and Y. Guan, "Robust unit commitment with wind power and pumped storage hydro," IEEE Trans. Power Syst., vol. 27, no. 2, pp. 800-810, May 2012. 
[8] L. Zhao and B. Zeng, "Robust unit commitment problem with demand response and wind energy," IEEE PES General Meeting, 2012.

[9] M.E. Khodayar, M. Shahidehpour, and L. Wu, "Enhancing the dispatchability of variable wind generation by coordination with pumped-storage hydro units in stochastic power systems," IEEE Trans. Power Syst., vol. 28, no. 3, pp. 2808-2818, Aug. 2013.

[10] H. Wu, M. Shahidehpour, A. Alabdulwahab, and A. Abusorrah, "Thermal generation flexibility with ramping costs and hourly demand response in stochastic security-constrained scheduling of variable energy sources," IEEE Trans. Power Syst., vol. 30, no. 6, pp. 2955-2964, Nov. 2015.

[11] D.R. Finley, “A minimax approach to cost variance investigation with imperfect parameter knowledge,” Decision Sciences, vol. 10, pp. 52-62, Dec. 1989.

[12] G.L. Shevlyakov and N.O. Vilchevski, "Minimax variance estimation of a correlation coefficient for $\varepsilon$-contaminated bivariate normal distribution- ns," Statistics \& Probability Letters, vol. 57, no. 1, pp. 91-100, Mar. 2012.

[13] R. Jiang, J. Wang, M. Zhang, and Y. Guan, “Two-stage minimax regret robust unit commitment,” IEEE Trans. Power Syst., vol. 28, no. 3, pp. 2271-2283, Aug. 2013.

[14] L. Wu and M. Shahidehpour "Accelerating the Benders decomposition for network-constrained unit commitment problems," Energy Systems, vol. 1, no. 3, pp. 339-376, Aug. 2010.

[15] B. Hu and L. Wu, "Robust SCUC considering continuous/discrete uncertainties and quick-start units: a two-stage robust optimization with mixed-integer recourse," IEEE Trans. Power Syst., 2015 (to appear).

[16] M.A. Duran and I.E. Grossmann, "An outer-approximation algorithm for a class of mixed-integer nonlinear programs," Math. Program, vol. 36, no. 3, pp. 306-339, 1986.

[17] P. Kesavan, R.J. Allgor, E.P. Gatzke, and P.I. Barton, "Outer approximation algorithms for separable nonconvex mixed-integer nonlinear programs," Math Program, pp. 517-535, 2004.

[18] B. Hu and L. Wu, "Robust SCUC with multi-band bus load uncertainty," IEEE Trans. Power Syst., 2015 (to appear).

[19] http://www.nyiso.com/public/index.jsp.

[20] J. Zhao, T. Zheng, and E. Litvinov, "Variable resource dispatch through do-not-exceed limit," IEEE Trans. Power Syst., vol. 30, no. 2, pp. 820-828, Mar. 2015. 